\title{
Uranus after Solstice: Results from the 1998 November 6 Occultation
}

\author{
Leslie A. Young \\ Southwest Research Institute, 1050 Walnut Street, Suite 426, Boulder, Colorado 80302 \\ E-mail: layoung@boulder.swri.edu
}

Amanda S. Bosh

Lowell Observatory, 1400 W. Mars Hill Road, Flagstaff, Arizona $86001-4499$ and Visiting Observer, Infrared Telescope Facility at Mauna Kea, Hawaii

Marc Buie

Lowell Observatory, 1400 W. Mars Hill Road, Flagstaff, Arizona 86001-4499

J. L. Elliot

Department of Earth, Atmospheric, and Planetary Science and Department of Physics, Massachusetts Institute of Technology, Cambridge, Massachusetts 02139-4307; Lowell Observatory, 1400 W. Mars Hill Road, Flagstaff, Arizona 86001-4499; and Visiting Observer, Infrared Telescope Facility at Mauna Kea, Hawaii

and

Lawrence H. Wasserman

Lowell Observatory, 1400 W. Mars Hill Road, Flagstaff, Arizona 86001-4499

Received July 7, 1999; revised June 25, 2001

\begin{abstract}
We observed a stellar occultation of thestar $U 149$ by $U$ ranusfrom Lowell Observatory and the IRTF on Mauna Kea on November 6, 1998. The temperatures derived from isothermal fits to the Lowell lightcurves are $116.7 \pm 7.9 \mathrm{~K}$ for immersion, and $124.8 \pm 15.5 \mathrm{~K}$ for emersion. The secular increase in temperature seen during the period 1977-1983 has reversed. Furthermore, the rate of decrease ( $\geq 1.2 \mathrm{~K} / \mathrm{yr}$ ) cannot be explained solely by radiative cooling. Although the temperature structure of Uranus' upper atmosphere may be related to seasonal effects (e.g., the subsolar latitude) or local conditions (e.g., diurnally averaged insolation), these observations suggest nonradiative influences on the temperature, such as adiabatic heating/cooling or thermal conduction. (c) 2001 Academic Press

Key Words: Uranus, atmosphere; occultations; atmospheres, structure; atmospheres, dynamics.
\end{abstract}

\section{INTRODUCTION}

Following the discovery of the Uranian ring system in 1977 (Elliot et al. 1977, Millis et al. 1977), Uranus was the subject of intense monitoring by stellar occultations between 1977 (Elliot and Dunham 1979) and 1983. This was aided by the passage of Uranus through the galactic plane, ensuring an ample supply of occultation stars. This coverage allowed an investigation of tem- peratures in the Uranian upper atmosphere as a function of time and latitude, through the modeling of the refractive defocusing of starlight by the Uranian atmosphere. Seven occultations of the atmosphere of Uranus were observed in the years 1977-1983, probing latitudes of $-30^{\circ}$ to $26^{\circ}$ (Baron et al. 1989). A temperature increase was first noted by French et al. (1983). The equivalent isothermal temperatures at 1-100 $\mu$ bar derived from Uranus occultations increased from $\sim 100 \mathrm{~K}$ in 1977 to $\sim 170 \mathrm{~K}$ in 1983, with a temporal increase of $\sim 8 \mathrm{~K} \mathrm{yr}^{-1}$ (Baron et al. 1989). No correlation with latitude was detected. (Note that for Uranus, the rotational north pole and the IAU definition of the north pole disagree. The rotational north pole is defined so that the planet rotates counterclockwise as viewed from above the rotational north pole. The IAU defines the north pole as the rotation axis that lies on the north side of the invariable plane of the solar system. To facilitate comparisons with earlier papers, latitudes in this paper are given relative to the rotational North pole of Uranus, following the notation in Baron et al. 1989 and Sicardy et al. 1985.)

All published occultations of the Uranian atmosphere at 1$100 \mu$ bar occurred before Uranus' southern summer solstice in 1985, except the Voyager UVS occultation in 1986 (Broadfoot et al. 1986, Herbert et al. 1987). Since Uranus' subsolar latitude changed from $51^{\circ}$ in 1977 to $76^{\circ}$ in 1983 , one major goal of 
our observations was to test whether the temperature increase was secular or cyclical. The subsolar latitude was $37^{\circ}$ at the time of the observations reported here (Standish et al. 1995), more equatorial than the first Uranian occultation in 1977. If the temperature variation were tied strictly to season, we would expect a temperature slightly less than that observed in 1977.

\section{OBSERVATIONS}

Uranus was predicted to occult the star U149 of the list of Klemola and Mink (1991) on 1998 Nov 6 UT with a sky-plane velocity of $17.61 \mathrm{~km} / \mathrm{s}$. We obtained observations at Lowell Observatory and at NASA's Infrared Telescope Facility (IRTF) on Mauna Kea, Hawaii (Fig. 1). L. A. Young, M. Buie, and L. Wasserman observed the occultation of U149 by Uranus using the PCCD camera (Buie et al. 1993) at the 72-inch Perkins telescope at Lowell Observatory's Anderson Mesa site in Flagstaff, Arizona. We collected data from 3:30 (Uranus at an altitude of $27^{\circ}$ ) to 5:47 UT, with the last useful data taken at about 5:30, when Uranus was at an altitude of $7.5^{\circ}$. The sky was photometric. In order to observe continuously for the two hours spanning the occultation without exhausting memory limitations, we used $3 \times 3$ on-chip binning, for an effective pixel size of 1.2 arcsec, a subframe readout with a box size of $20 \times 20$ binned pixels $(60 \times 60$ unbinned pixels), and a data rate of $250 \mathrm{~ms}$ per expo-

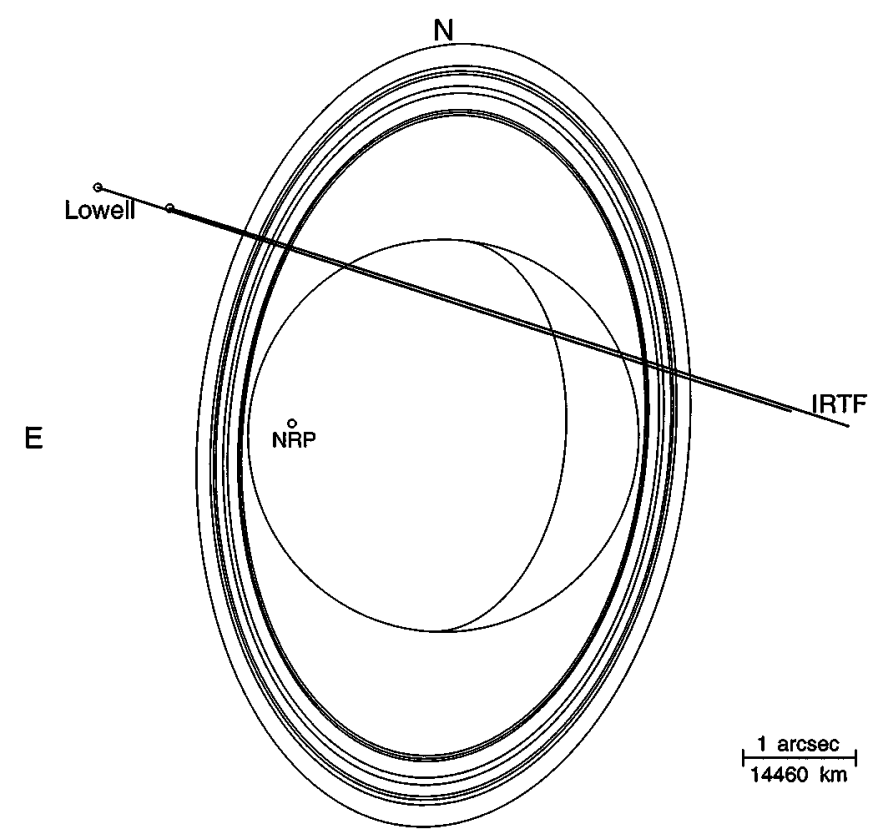

FIG. 1. Uranus and its rings as seen from a topocentric observer at the midtime of the event. Also shown are the tracks of the star as seen from the IRTF and Lowell Observatory, with circles indicating the beginnings of the drawn tracks. The track for the IRTF is slightly north of the track for Lowell because Uranus' topocentric velocity had a significant northward motion during the event. The north rotational pole (NRP) is indicated; this is the same as the IAU south pole. sure. We observed with a methane filter at $0.89 \mu \mathrm{m}(0.10 \mu \mathrm{m}$ FWHM), to decrease the contribution from Uranus. Through this filter, Uranus was six times brighter than U149, a factor of 22 improvement over the contrast through a Kron-Cousins R filter. A. S. Bosh and J. L. Elliot observed the emersion event using the NSFCAM at the IRTF. Immersion was not observed, because of clouds that cleared before the emersion ring occultations. Unfortunately, remaining thin clouds rendered the IRTF background levels too unstable for deriving atmospheric temperatures. The remainder of this paper discusses the Lowell observations.

\section{ANALYSIS}

Because of the large pixel size used at Lowell, we were not able to resolve Uranus and U149 during the event. We performed synthetic aperture photometry on the bias-subtracted images using a circular aperture with a radius of four binned pixels $(4.9$ arcsec). The sky was estimated from a region that extended to the edges of the subframe and had an inner radius of seven pixels ( 8.61 arcsec). We used the upper baselines just before atmospheric immersion or just after atmospheric emersion to calculate which synthetic aperture would maximize the signal-to-noise ratio (SNR). From these tests, the SNR per point of the total flux (Uranus + U149) was 56 for immersion, and 20 for emersion. The quality of an occultation can be characterized by a single dimensionless measure of the noise, $\left(\nu_{\perp} / H\right)^{1 / 2} \varepsilon$, where $v_{\perp}$ is the apparent velocity of the star perpendicular to the planetary limb, $H$ is the scale height, and $\varepsilon$ is the noise per second of the nomalized flux (French et al. 1978). For U149, the noise measures were rather high compared with other Uranian occultations (see French et al. 1983): 0.023 for immersion and 0.044 for emersion, low enough for isothermal fitting, but too large for direct inversion.

We were able to model the upper and lower baselines of the entire two hours of observations (Fig. 2) with parameters describing the flux of Uranus at mid-event, the magnitude difference of the star and Uranus, an extinction coefficient, and three parameters for a low-altitude equation for airmass valid for altitudes as small as $6^{\circ}$ (Snell and Heiser 1968). In this case, we find the airmass, $X$, was well described by $X=X^{\prime}-0.0018\left(X^{\prime}-\right.$ 1) $-0.0029\left(X^{\prime}-1\right)^{2}-0.0008\left(X^{\prime}-1\right)^{3}$, where $X=$ csc (altitude) is the usual (high-altitude) expression for the airmass. To determine the lower baseline while avoiding contamination by residual stellar flux at the $1 \%$ level, we excluded the data obtained less than 10 minutes after the immersion half-light time or less than 10 minutes before the emersion half-light time. The careful removal of the upper and lower baselines was important because of the rapidly changing airmass during emersion, leading to baselines that changed nonlinearly with time.

The geometry of the event was determined using the pole position and ring parameters given by French et al. (1991) and fitting for the star position offset using the ring geometry method of Bosh (1994). The specific Uranus ring-plane pole, gravitational harmonic parameters, star position and fitted offset, and ring 


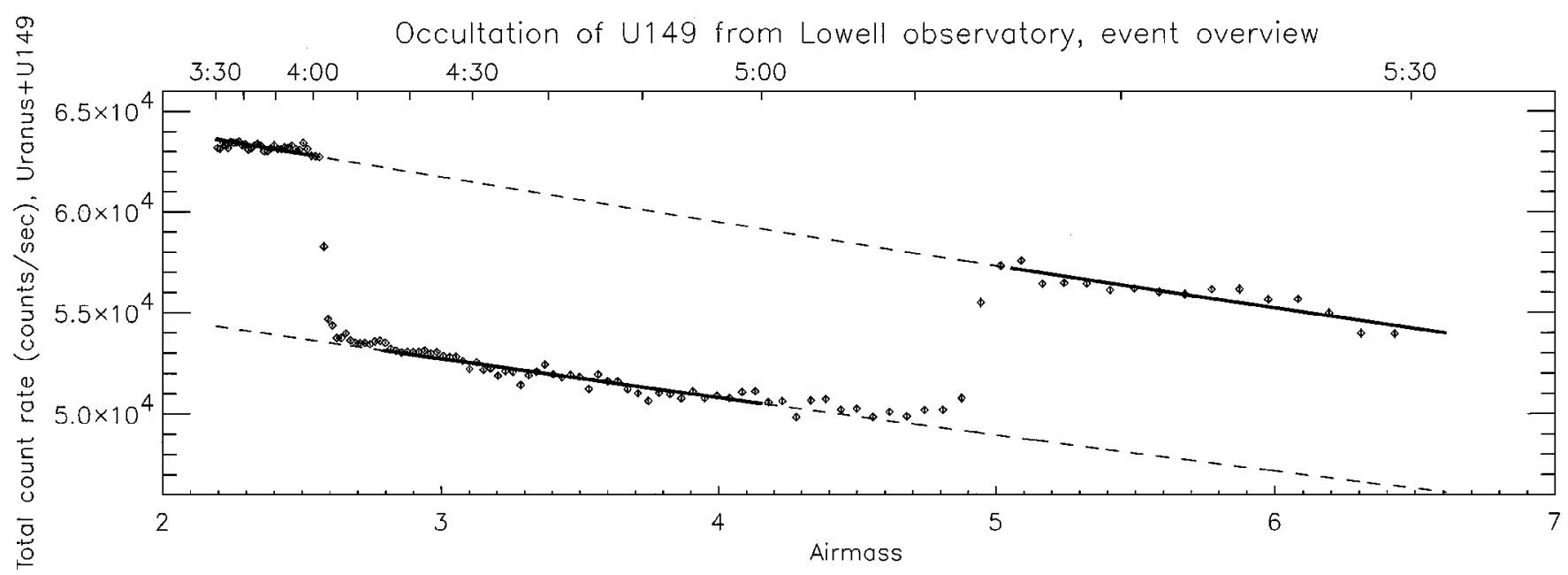

FIG. 2. Overview of event, binned to 1-minute averages. Thick lines indicate the airmasses included in the fits for the airmass correction and baseline estimates. At this binning, the narrow rings are not visible.

feature times are listed in Table I. The planet ephemeris used was DE406. For the geometric fit, we included data from both Lowell and the IRTF; the varying background levels in the IRTF data did not affect the timing of ring features. We used only those ring features that were described by French et al. (1991) as having a simple eccentric orbit model, with $m=1$; therefore, we did not include the $\delta$ or $\gamma$ ring timings in this analysis. In our nonlinear least-squares fits to the observed ring feature times, we minimized the residual in ring-plane radius. In this fit, all features were weighted equally. The RMS residual per degree of freedom was $4.6 \mathrm{~km}$ for the Lowell and IRTF data together. The value of the IRTF data for this event is that the timing of the ring observations allowed us to establish the shadow-plane radius as a function of time from the rings alone, independently from the atmospheric analysis.

The scale heights $(H)$ and half-light shadow radii $\left(\rho_{\mathrm{h}}\right.$, the distance between the observer and the center of the planet's shadow when the stellar flux is half the unocculted value) were found by fitting large-planet, isothermal models (Baum and Code 1953) of normalized flux as a function of shadow radius (Table II; Fig. 3). The upper and lower baselines were also allowed to be free parameters, but were within $1 \%$ of their expected values. Correcting for gravitational and refractive bending and for the effects of an oblate planet (Baron et al. 1989), we find the half-light of the occultation occurred at a latitude of $27.30^{\circ}$ for immersion and $-43.29^{\circ}$ for emersion, at planet radii $\left(r_{\mathrm{h}}\right)$ of $25952.7 \pm 7.6$ and $25832.8 \pm 14.8 \mathrm{~km}$, consistent with the half-light surface predicted by the oblateness model of Baron et al. (1989). The half-light latitudes of previously published occultations spanned $-29.9^{\circ}$ to $25.8^{\circ}$ (Baron et al. 1989), so these observations extend the range of latitudes observed. As Uranus' aspect becomes more equatorial, we can expect occultations that probe a wider range of latitudes, including previously unsampled higher latitudes. This will allow a more accurate measure of the shape of the constant refractivity surface near $1 \mu$ bar.

To convert scale height to temperature, we use the mean molecular weight of 2.324 amu inferred by Conrath et al. (1987) from Voyager radio occultations and infrared spectroscopy measurements, corresponding to a helium mole fraction of $0.152 \pm$ 0.033 . The refractivity at STP at $8900 \AA$ for this mixture is $1.215 \times 10^{-4}$ (Peck and Huang 1977, Mansfield and Peck 1969). The effective gravity, $g$, was computed using $G M=$ $5,793,939 \mathrm{~km}^{3} \mathrm{~s}^{-2}$ (Tyler et al. 1986), harmonic coefficients defined at $R=26,200 \mathrm{~km}$ of $J_{2}=3.34343 \times 10^{-3}$ and $J_{4}=$ $-2.885 \times 10^{-5}$ (French et al. 1988), and a rotation period at the $1 \mu$ bar level of $17.7 \mathrm{hr}$ derived from Uranus' oblateness by Baron et al. (1989), yielding $g=8.48$ and $8.52 \mathrm{~m} \mathrm{~s}^{-2}$ for immersion and emersion, respectively. Because a primary goal of this analysis is the comparison with previous observations, no attempt was made to account for the vertical variation of gravity or molecular weight. The equivalent isothermal temperature for this event was $116.7 \pm 7.9 \mathrm{~K}$ for immersion and $124.8 \pm$ $15.5 \mathrm{~K}$ for emersion. An attempt was made to fit simultaneously for a scale-height gradient and scale height (Elliot and Young 1992) and to invert the data, but the data did not have a sufficiently high signal-to-noise ratio (SNR).

The half-light planet radii were converted to radii in the shadow plane using the fitted scale height and the calculated bending resulting from general relativity. The astrometric solution then allowed us to calculate the times of half light. The number density and pressure at half light are derived from the half-light planet radius and the scale height (e.g., Baum and Code 1953) using a refractivity at standard temperature and pressure, $v_{\mathrm{STP}}$, of $1.215 \times 10^{-4}$ (at $0.89 \mu \mathrm{m}$ for a He mole fraction of 0.152; Allen 1976).

When the temperature derived from isothermal fits to occultation lightcurves are interpreted as indicative of the temperatures 
TABLE I

Geometric Model Parameters

Uranus ring-plane pole (French et al. 1991)

$\alpha(\mathrm{deg}, \mathrm{J} 2000)$
$\delta(\mathrm{deg}, \mathrm{J} 2000)$
Epoch
$\partial \alpha / \partial t\left(\operatorname{deg} \mathrm{yr}^{-1}, \mathrm{~J} 2000\right)$
$\partial \delta / \partial t\left(\operatorname{deg} \mathrm{yr}^{-1}, \mathrm{~J} 2000\right)$

76.5969

15.1117

$\mathrm{J} 2000$

0.0

0.0

Gravitational harmonic coefficients (French et al. 1988)

\begin{tabular}{cr}
\hline$J_{2}$ & 0.00334343 \\
$J_{4}$ & -0.00002885 \\
$J_{6}$ & 0.0 \\
Equatorial radius & $26200 \mathrm{~km}$ \\
\hline
\end{tabular}

Star position (Klemola and Mink 1991)

\section{Name}

Catalog $\alpha(\mathrm{J} 2000)$

Catalog $\delta(\mathrm{J} 2000)$

$\alpha_{0}(\operatorname{arcsec}, \mathrm{J} 2000)$

$\delta_{0}(\operatorname{arcsec}, \mathrm{J} 2000)$

Adjusted $\alpha$ (J2000)

Adjusted $\delta$ (J2000)

Feature times

Time (UTC on 6 Nov. 1998)

$\begin{array}{rc}\text { Feature } & \text { Lowell } \\ \text { Ingress } \varepsilon & 33913.5 \\ \delta & 34218.0^{a} \\ \gamma & 34307.0^{a} \\ \eta & \\ \beta & 34533.0 \\ \alpha & 34639.0\end{array}$

51156.0

51228.0

51506.0

51618.0

51812.0

$51845.0^{a}$

$51936.0^{a}$

52845.0

${ }^{a}$ These features not used in the geometric fit.

near the half-light level, or pressures near 1-10 $\mu$ bar, they appear to grossly underestimate the temperatures derived from UVS occultations (e.g. Strobel et al. 1991 for Uranus, and Yelle et al. 1996 for Jupiter). The discrepancy, however, lies not in the temperature, but in the pressure assigned to that temperature. This is easily demonstrated for the case of an atmosphere with a constant temperature gradient for which the scale height is given by $H=H_{h}+H^{\prime}\left(r-r_{h}\right)$, where $H$ is the scale height,
$H_{h}$ is the scale height at the half-light radius, and $H^{\prime}$ is the scale height gradient. For $H^{\prime} \ll 1$, such an atmosphere would produce a lightcurve which is similar in shape to one made by an atmosphere with a constant scale height, $H_{\text {iso }}=H_{h} /\left(1+(5 / 2) H^{\prime}\right)$ (Elliot and Young 1992), with identical slopes at half-light. (Note that the factor of $5 / 2$ is a correction to an earlier derivation of $3 / 2$ by Goldsmith (1963)). By eliminating $H_{h}$, we find that $H=H_{\text {iso }}$ when $r=r_{h}-(5 / 2) H_{i s o}$. Therefore, the equivalent isothermal temperature equals the actual temperature at 2.5 scale heights below the half-light level.

The above analysis cannot be applied directly to giant-planet atmosphere near 1-100 $\mu$ bar, where the derivatives in the scale height are large and change with height. To address the gradient problem in realistic atmospheres, we apply the error analysis of French et al. (1978). We caution that the French et al. (1978) analysis was based on the inversion method of occultation analysis, rather than forward modeling, so the following discussion has yet to be put on a firm mathematical foundation.

The normalized flux decreases as an atmospheric occultation proceeds, lowering the SNR for a single datum. Meanwhile, because of the differential refraction, equally spaced intervals in altitude get mapped to increasingly larger intervals in time. With more data points per altitude interval, this tends to increase the SNR. For measurements of the temperature from unweighted or equally weighted data, French et al. (1978) find that these two competing effects lead to a minimum in the temperature's fractional error 2.6 scale heights below half-light. This is in agreement with the mild-gradient formula of Elliot and Young (1992), and with the numerical tests of mild gradients performed by Wasserman and Veverka (1973). For the case at hand, the relevant question is the relevance of isothermal fits, given the complex thermal structure seen in the Uranian atmosphere between 1 and $100 \mu$ bar. To answer this, we performed isothermal fits to lightcurves generated from the Voyager Ultraviolet Spectrometer (UVS) thermal profile (Herbert et al. 1987). Figure 4 clearly demonstrates that, for realistic atmospheres, the equivalent isothermal temperature is a good measure of the temperature 2.6 scale heights below the half-light level. Furthermore, for previous observations of Uranian occultations, the temperatures derived from isothermal fits agreed well with temperatures below half-light derived from inversions (French et al. 1982, French et al. 1987, Millis et al. 1987) Therefore, we believe the trends in temperature noted by Baron et al. (1989) reflect real changes in the temperature at 10 to $30 \mu$ bar.

Are the temperature variations entirely the result of spatial, not temporal, inhomogeneity? Although multiple observations during a single occultation show that there is spatial variation in temperature of $\sim 40 \mathrm{~K}$ over $30^{\circ}$ of latitude (from thermal inversion; Sicardy et al. 1985), there is a spread of $70 \mathrm{~K}$ for occultations taken at the same latitude, at different times (using isothermal model fits; Baron et al. 1989). This is seen as well in Fig. 5a, where the temperatures for latitudes $-20^{\circ}$ to $-15^{\circ}$ 
TABLE II

Summary of Occultation Results

\begin{tabular}{|c|c|c|c|c|c|c|c|c|c|}
\hline Event & $\begin{array}{l}\text { Half-light time } \\
t_{\mathrm{h}}(\mathrm{UT})\end{array}$ & $\begin{array}{c}v_{\perp} \text { at half-light } \\
(\mathrm{km} / \mathrm{s})\end{array}$ & $\begin{array}{c}\text { Scale Height } \\
H(\mathrm{~km})\end{array}$ & $\begin{array}{c}\text { Shadow radius } \\
\rho_{h}(\mathrm{~km})\end{array}$ & $\begin{array}{c}\text { Planet radius } \\
r_{h}(\mathrm{~km})\end{array}$ & $\begin{array}{l}\text { Half-light density } \\
n_{h}\left(10^{13} \mathrm{~cm}^{-3}\right)\end{array}$ & $\begin{array}{l}\text { Half-light pressure } \\
\qquad p_{h}(\mu \mathrm{bar})\end{array}$ & $\begin{array}{l}\text { Latitude } \\
\qquad \theta\left({ }^{\circ}\right)^{a}\end{array}$ & $\begin{array}{c}\text { Temperature } \\
T(\mathrm{~K})\end{array}$ \\
\hline Im & $4: 02: 27.17 \pm 0.98$ & -6.958 & $49.7 \pm 3.4$ & $25870.2 \pm 6.8$ & $25952.7 \pm 7.6$ & $6.44 \pm 0.66$ & $1.04 \pm 0.18$ & 27.30 & $116.7 \pm 7.9$ \\
\hline Em & $5: 13: 14.32 \pm 1.89$ & 7.025 & $54.4 \pm 6.5$ & $25740.7 \pm 13.3$ & $25832.8 \pm 14.8$ & $7.39 \pm 1.32$ & $1.27 \pm 0.40$ & -43.29 & $124.8 \pm 15.5$ \\
\hline
\end{tabular}

${ }^{a}$ Latitudes are defined relative to the rotational north pole.

and $+4^{\circ}$ to $+9^{\circ}$ both show strong temporal variation. Baron et al. (1989) plot temperature versus latitude and show no systematic trend. Although there undoubtedly are spatial variations in the Uranian atmosphere, there must be temporal variations as well.

While French et al. (1983) and Baron et al. (1989) interpreted the temperature increase as a suggestion that the Uranian middle atmosphere tends to heat and cool as a whole, these earlier observations were limited to latitudes between $-30^{\circ}$ and $20^{\circ}$. Probes of the middle atmosphere by the Voyager UVS at higher latitudes indicate latitudinal variability at the 0.1 to
$1 \mu$ bar level (Herbert et al. 1987), leading Strobel et al. (1991) to suggest radiatively driven adiabatic cooling of the sunlit hemisphere. Another interesting possibility is that we are seeing diurnal weather patterns. By coincidence, Uranus passed south of the occulted star for the event reported here and all those reported in Baron et al. (1989). Therefore, the presolstice events reported in Baron et al. (1989) probed the dawn limb, while this event probed the dusk limb. The observation and analysis of occultations that are closely spaced in time, but widely spaced in latitude, may help to separate latitudinal, seasonal, and diurnal variations.

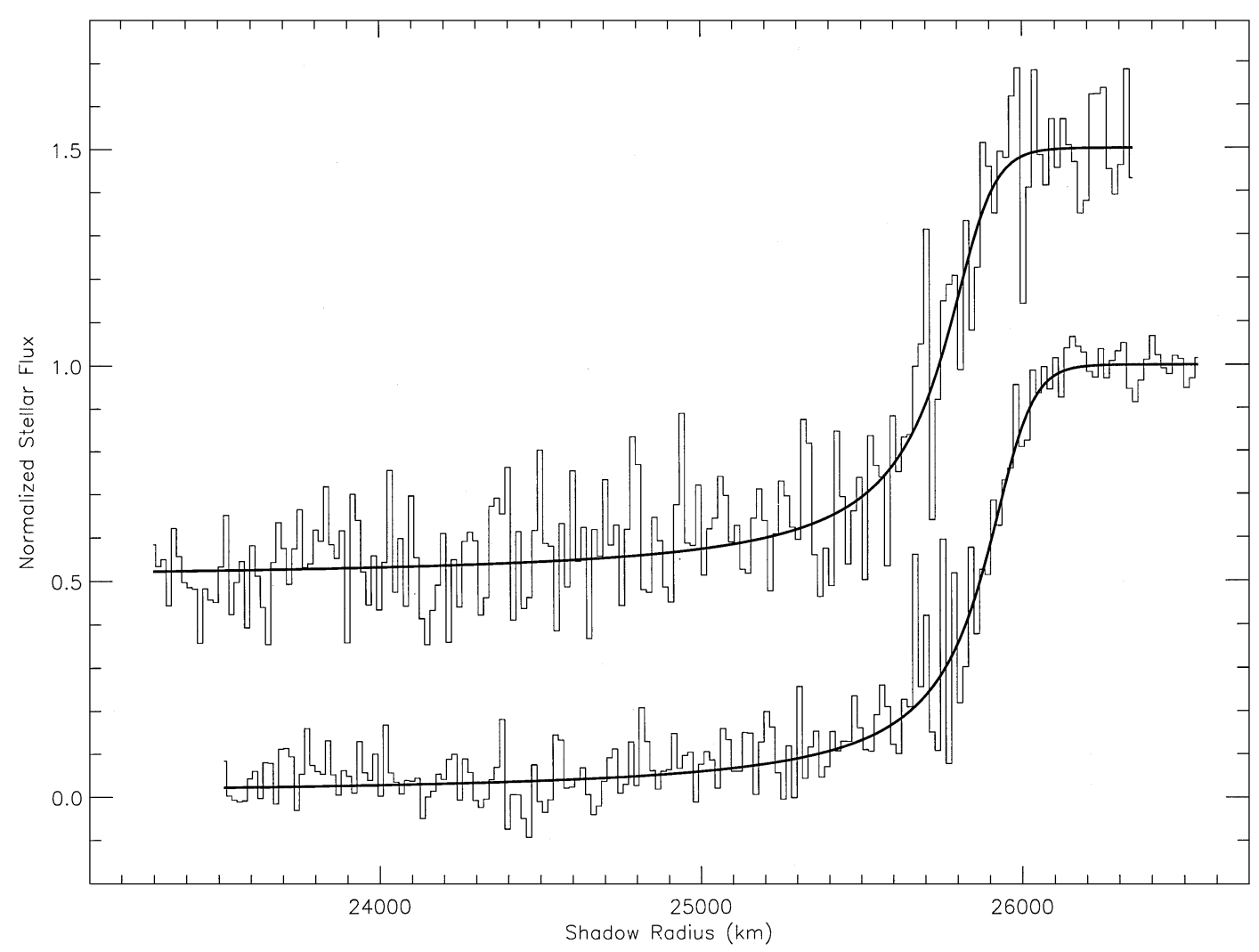

FIG. 3. Normalized stellar flux during immersion (lower) and emersion (upper, offset by 0.5 ) as a function of distance from the center of the occultation shadow, for the 1998 November 6 occultation observed from Lowell Observatory. The smooth, thick lines are the best-fit isothermal lightcurves, as described in the text and in Table II. The data are shown as thin lines. To aid the comparison of data and model, the data have been binned by a factor of 10 ( $2.5 \mathrm{~s}$ per plotted point). The fits were performed on the unbinned data. The increase in the residuals during the main drop is caused by scintillation spikes from small-scale density fluctuations in the Uranian atmosphere. 

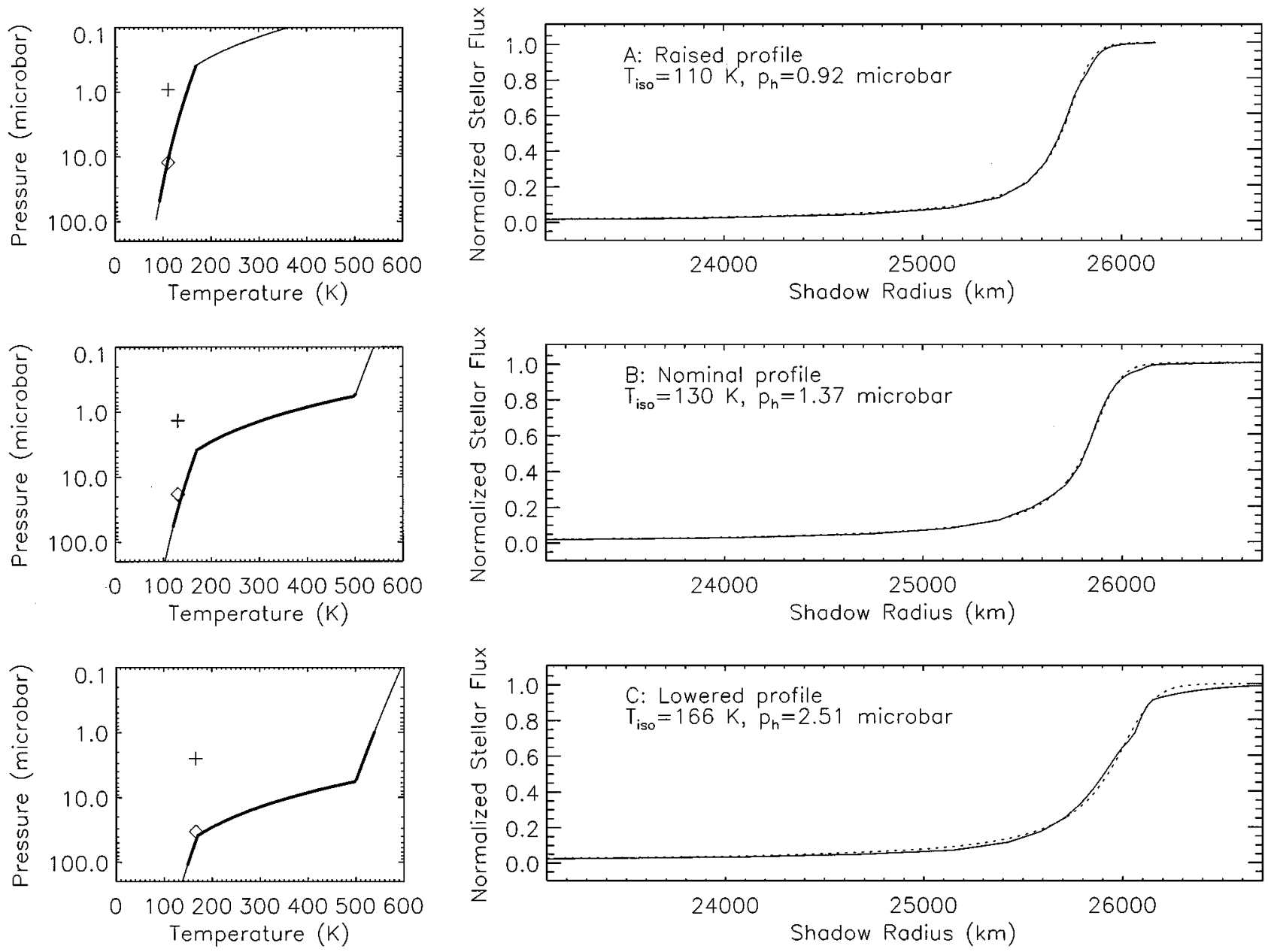

FIG. 4. Isothermal fits to realistic atmospheres. Three representative nonisothermal atmospheres are plotted on the left-hand column. The middle panel (model B) is the "compromise" atmosphere of Herbert et al. (1987), while the pressures in models A and C have been arbitrarily divided or multiplied by a factor of 10. The synthetic occultation lightcurve for each atmosphere is plotted as a solid line on the right-hand column, with the best-fit isothermal lightcurve plotted as a dashed line. Notice the similarity of the two curves, especially for models A and B. On the left-hand plots, the thick line indicates the region corresponding to one scale height above half-light to four scale heights below. The plus marks the equivalent isothermal temperature $\left(T_{i s o}\right)$ at the half-light pressure $\left(p_{h}\right)$, while the diamond marks $T_{i s o}$ at 2.6 scale heights below half-light. Note the close agreement of the lower point with the original atmospheric profile in all three cases.

\section{DISCUSSION}

\subsection{Heating Sources}

Stellar occultations are most sensitive to a region spanning roughly one scale height above the half-light level to four scale heights below (French et al. 1978), corresponding in this case to pressures of $\sim 0.3$ to $55 \mu \mathrm{bar}$, or number densities of $2 \times 10^{13}$ to $3 \times 10^{15} \mathrm{~cm}^{-3}$. Radiative-convective models of the Uranian upper atmosphere generally assume that the temperature is determined by the balance between radiative heating by $\mathrm{CH}_{4}$ in the near infrared and cooling by hydrocarbons, including $\mathrm{CH}_{4}$ at $7.7 \mu \mathrm{m}$ (Appleby 1986, Appleby 1990, Marley and McKay 1999). However, several lines of evidence suggest that absorption of sunlight by hydrocarbons in the near infrared is not the only source of heating in the Uranian atmosphere at these pressures.
The first line of evidence comes from a comparison of observed temperatures to those predicted by radiative equilibrium models, which find the temperatures needed for the radiative heating and cooling rates to balance. French et al. (1983) estimated the heating rate in the $3.3 \mu \mathrm{m}$ band of $\mathrm{CH}_{4}$ to be only $0.3 \mathrm{~K} \mathrm{yr}^{-1}$, for a $\mathrm{CH}_{4}$ mixing ratio of $3 \times 10^{-5}$ (from Appleby 1980) and a normalized diurnally averaged insolation of $1 / 4$. Using the same mixing ratio, Sicardy et al. (1985) found that absorption of solar UV flux by $\mathrm{CH}_{4}$ between 1000 and $1500 \AA$ dominated over IR heating at pressures lower than 5-10 $\mu$ bar, for the occultation geometries observed to date. The radiative cooling rates are strongly temperature dependant; French et al. (1983) calculated radiative cooling rates at a variety of number densities and temperatures as a result of $\mathrm{CH}_{4}$ (at a mixing ratio of $3 \times 10^{-5}$ ) and $\mathrm{C}_{2} \mathrm{H}_{2}$ (at a mixing ratio of $2 \times 10^{-8}$ ), and found cooling rates at $10^{14} \mathrm{~cm}^{-3}$ that ranged from $0.066 \mathrm{~K} \mathrm{yr}^{-1}$ 

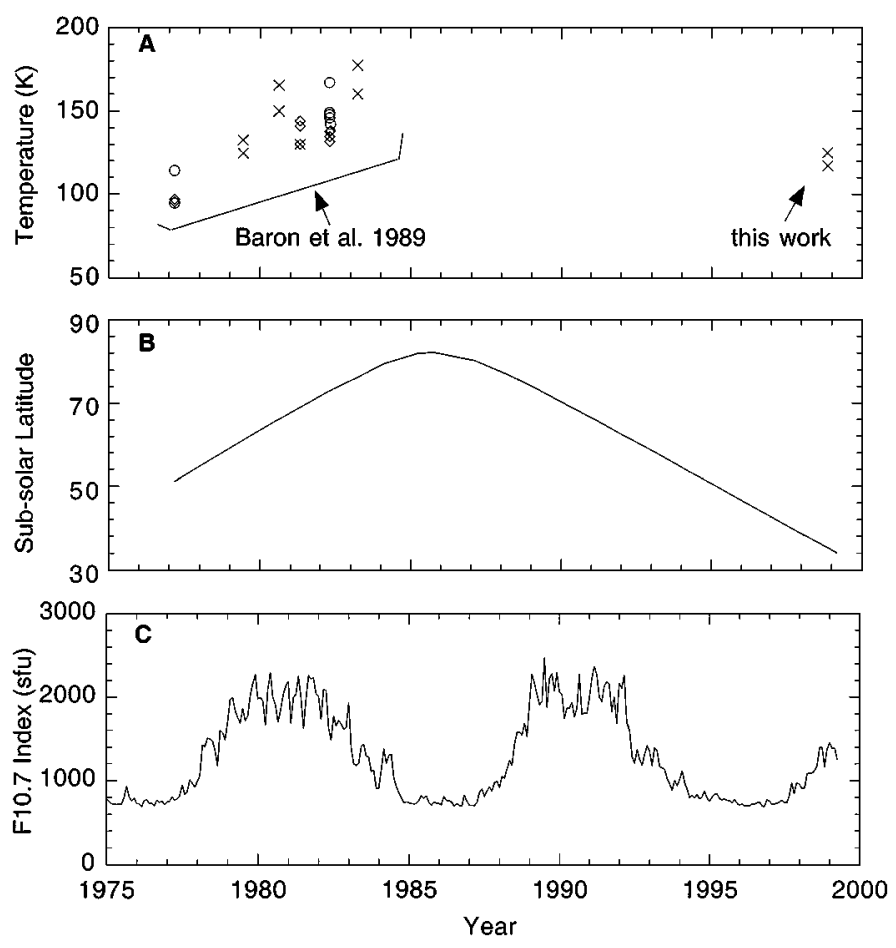

FIG . 5. (a) Equivalent isothermal temperature versus date of observation, from Baron et al. (1989) and this work. The temperatures in Baron et al. (1989) have been adjusted to use the values of mean molecular weight and gravitational harmonic coefficients described in the text. Latitudes range from $-34^{\circ}$ to $+27^{\circ}$ with most of the measurements falling within two narrow latitude bands: $-20^{\circ}$ to $-15^{\circ}$ (diamonds) and $+4^{\circ}$ to $+9^{\circ}$ (circles). All other latitudes are plotted as crosses. Baron et al. do not provide error bars for temperatures, but from their discussion of errors for half-light times and the scaling of French et al. (1978), we estimate these measurements have errors typically around 1-5 K. (b) Subsolar latitude (defined with the rotational North pole at $+90^{\circ}$ ) vs. date. (c) The solar f10.7 index versus date, a proxy for solar UV activity (Covington 1969).

at $100 \mathrm{~K}$ to $71.4 \mathrm{~K} \mathrm{yr}^{-1}$ at $175 \mathrm{~K}$. Similarly, Sicardy et al. (1985) found cooling rates of $\sim 10 \mathrm{~K} \mathrm{yr}^{-1}$ at $140 \mathrm{~K}$. A detailed nonLTE calculation by Appleby (1990) found radiative-equilibrium temperatures at the 1 to $100 \mu$ bar region of $120-125 \mathrm{~K}_{\text {for }} \mathrm{CH}_{4}$ mixing ratio of $2 \times 10^{-5}$. Thus, all observed temperatures except those from 1977 (Fig. 5a) are warmer than expected from radiative equilibrium.

The heating rates described above relied on pre-Voyager estimates of the hydrocarbon mixing ratios. With the Voyager UVS measurements of $\mathrm{CH}_{4}$ in the upper atmosphere (Herbert et al. 1987, Bishop et al. 1990), the discrepancy between observed and predicted temperatures has widened. Marley and McKay (1999) recently applied a radiative-convective model to the Uranian atmosphere, using $\mathrm{CH}_{4}$ abundances at $1 \mu$ bar that range from $10^{-10}$ to $10^{-7}$, spanning the UVS stellar occultation value of $6 \times 10^{-9}$. Their predicted temperatures are significantly lower than those observed between 10 and $100 \mu$ bar. In particular, the equilibrium temperature at $30 \mu$ bar for the nominal UVS abundance is predicted to be less than $100 \mathrm{~K}$.
The second line of evidence for additional heating sources comes from the presence of large-scale features in the thermal profiles derived from the inversion of stellar occultations (French et al. 1983). Thermal conduction acts to elimintate temperature contrasts. French et al. (1983) estimate that a heating rate of $1-10 \mathrm{~K} \mathrm{yr}^{-1}$ is required to maintain the large nonisothermal structures observed in the Uranian upper stratosphere in the face of thermal conduction.

The final line of evidence comes from the temperature rise derived from stellar occultations observed between 1977 and 1983, which requires a heating source capable of changing the temperature by $\sim 8 \mathrm{~K} /$ year (Fig. 5a; Baron et al. 1989, French et al. 1983).

Suggestions for the additional heating term have included absorption of sunlight by $\mathrm{CH}_{4}$ in the ultraviolet (Sicardy et al. 1985), radiation propagating downward from a hot thermosphere (Marley and McKay 1999), heating by infalling dust (Rizk and Hunten 1990), deposition of energy carried by dissipating gravity waves (French et al. 1983), and Joule heating, which arises from the collisions between ions and the neutral atmosphere (Herbert et al. 1987).

\subsection{Cooling by Radiation}

As shown in Fig. 5a, the heating trend observed between 1977 and 1983 has reversed. We have already argued for the presence of an unknown nonradiative heating source. Can the net cooling between 1983 and 1998 be explained simply as the result of the lessening of this heating source? Initially, it seems plausible that the heating source may be tied to the subsolar latitude (Fig. 5b) or the solar cycle (Fig. 5c). However, we show below that the radiative cooling rates are far too slow to explain the observed decrease in temperature.

A radiative-convective, seasonal model of the Uranian atmosphere is beyond the scope of this paper. Rather, we show the inadequacy of radiative cooling analytically, even for the most favorable case. For the hydrocarbons to radiate as efficiently as possible, we assume that the methane, ethane, and acetylene bands are optically thin, and in local thermodynamic equilibrium. In addition, we make the flagrantly unrealistic assumption that there is no source of heating to balance the radiative cooling.

Under these extreme assumptions, the cooling rate can be expressed as $d T / d t=-a \xi e^{-b / T}$, where $\xi$ is the mole fraction of the radiator, $a=8 \pi S h v^{3} /\left(c^{2} m c_{p}\right)$, and $b=h v / k$. Here $S$ is the band strength in $\mathrm{Hz}$ molecule ${ }^{-1} \mathrm{~cm}^{2}, c$ is the speed of light, $m$ is the mean mass of a molecule, $c_{p}$ is the specific heat at constant pressure $\left(1.45 \times 10^{8}\right.$ erg $\left.\mathrm{K}^{-1} \mathrm{~g}^{-1}\right), h$ is Planck's constant in erg s, $v$ is the frequency of the transition in $\mathrm{Hz}$, and $k$ is Boltzmann's constant in erg $\mathrm{K}^{-1}$. Using the 1996 edition of the HITRAN database (Rothman et al. 1992), we find $a=1.11 \times 10^{11}, 7.66 \times 10^{9}$, and $8.27 \times 10^{10} \mathrm{~K} \mathrm{yr}^{-1}$ and $b=$ 1868.1, 1169.5, and 1034.8 $\mathrm{K}$ for $\mathrm{CH}_{4}, \mathrm{C}_{2} \mathrm{H}_{6}$, and $\mathrm{C}_{2} \mathrm{H}_{2}$, respectively. For the mole fractions considered in Marley and McKay (1999), the radiative cooling rate at $30 \mu$ bar is dominated by the 
$7.7 \mu \mathrm{m}$ band of $\mathrm{CH}_{4}$. In the case of cooling by a single band, the cooling rate can be explicitly integrated to give the time, $\Delta t$, required to cool from an initial temperature $T_{i}$ to a final temperature $T_{f}$,

$$
\Delta t=\frac{\left(e^{b / T_{f}}-e^{b / T_{i}}\right)-b\left(E i\left(b / T_{f}\right)-E i\left(b / T_{i}\right)\right)}{a \xi},
$$

where $E i$ is the exponential integral (Abramowitz and Stegun 1964).

Two events were observed in early 1983, with equivalent isothermal temperatures of 177.4 and $163.5 \mathrm{~K}$ (Baron et al. 1989). The two events reported here, observed in late 1998, had equivalent isothermal temperatures of $116.7 \pm 7.9$ and $124.8 \pm$ $15.0 \mathrm{~K}$. The nominal drop is therefore from $\sim 170$ to $\sim 122 \mathrm{~K}$, for a nominal rate of $3 \mathrm{~K} \mathrm{yr}^{-1}$. Again, to be extremely conservative, we decrease the temperature difference by $15 \mathrm{~K}$ on either extreme, and ask how long it would take to cool the atmosphere from $155 \mathrm{~K}$ to $137 \mathrm{~K}$. Using the $\mathrm{CH}_{4}$ mixing ratio of $1 \times 10^{-6}$ at $30 \mu$ bar assumed by Marley and McKay (1999), we find that even the most optimistic cooling rates would require over 4000 years to produce the observed decrease in temperature. In order to produce the observed drop in only 15.6 years would require a methane mole fraction of at least $5 \times 10^{-5}$. There is evidence that the pressure of Jupiter's homopause changes with solar cycle (Wolven and Feldman 1998), and presumably Uranus's homopause can change as well, leading to variable mole fractions at $30 \mu \mathrm{bar}$. If the stratospheric methane mole fraction is $10^{-4}$, as assumed by Marley and McKay (1999), the eddy diffusion coefficient would need to change by an order of magnitude in order to bring enough $\mathrm{CH}_{4}$ up to the occultation pressure levels (Herbert et al. 1987). If the stratospheric methane mole fraction is only slightly less, then no amount of eddy mixing can supply the methane required to cool the atmosphere by radiation through its $7.7-\mu \mathrm{m}$ band.

At 100 to $175 \mathrm{~K}$, two $\mathrm{CH}_{4}$ molecules are less efficient radiators than a single molecule of either $\mathrm{C}_{2} \mathrm{H}_{2}$ or $\mathrm{C}_{2} \mathrm{H}_{6}$ by two to three orders of magnitude, raising the possibility that changes in the solar cycle can increase the photochemical production of $\mathrm{C}_{2} \mathrm{H}_{2}$ or $\mathrm{C}_{2} \mathrm{H}_{6}$, leading to an increased radiative cooling rate. Again, we ask how large a mole fraction is required to decrease the temperature from 155 to $137 \mathrm{~K}$ in 15.6 years, and find minimum mole fractions of $1.4 \times 10^{-7}$ for $\mathrm{C}_{2} \mathrm{H}_{2}$ or $3.8 \times 10^{-6}$ for $\mathrm{C}_{2} \mathrm{H}_{6}$. From the Voyager 2 UVS occultations, Bishop et al. (1990) inferred molar fractions of only $10^{-12}$ for ethane and acetylene at $30 \mu \mathrm{bar}$, more than seven orders of magnitude too small. Since the photochemically produced $\mathrm{C}_{2} \mathrm{H}_{2}$ and $\mathrm{C}_{2} \mathrm{H}_{6}$ should vary by less than a factor of 3 between solar minimum and solar maximum (Moses et al. 2000), photochemistry alone cannot produce a sufficiently large radiative cooling rate. This conclusion is supported by Marley and McKay (1999), who show that changing the $\mathrm{C}_{2} \mathrm{H}_{2}$ and $\mathrm{C}_{2} \mathrm{H}_{6}$ abundances by a factor of 10 does not affect the temperatures at 1-100 $\mu$ bar. The UVS profiles of ethane and acetylene peak at $\sim 200 \mu \mathrm{bar}$, with mole fractions that are slightly less than $10^{-7}$. Again, to bring the large stratospheric mixing ratios upward to the occultation level would require an order-of-magnitude increase in the eddy diffusion coefficient (Herbert et al. 1987).

Cooling from $\mathrm{H}_{3}^{+}$emission probably originates from higher altitudes than those probed by the occultation (Trafton et al. 1999; Encrenaz et al. 2000a, 2000b). The cooling rate from the CO rotational transitions can be expressed as $d T / d t=-a \xi T^{b}$, where $a=0.506 \mathrm{~K} \mathrm{yr}^{-1}$ and $b=2.134$, and $\xi$ is again the mole fraction of the emitter. In analogy to the rotation-vibration lines, we can integrate this to find the time required to cool from an initial temperature $T_{i}$ to a final temperature $T_{f}$.

$$
\Delta t=\frac{1}{a \xi(b-1)}\left(\frac{1}{T_{f}^{b-1}}-\frac{1}{T_{i}^{b-1}}\right) .
$$

For $\xi_{\mathrm{CO}}=3 \times 10^{-8}$ (Marley and McKay 1999), $\Delta t$ is longer than 28,000 years.

The above discussion strongly suggests nonradiative cooling mechanisms in the Uranian upper atmosphere, in addition to the nonradiative heating mechanisms already inferred. This mechanism is currently unknown, but below we briefly consider two candidates: the loss of energy to the stratosphere through thermal conduction, and adiabatic cooling resulting from a small vertical wind.

\subsection{Cooling by Conduction}

One way to remove energy from the upper atmosphere is by conduction to the lower atmosphere. To cool the entire occultation region (from $p_{\text {low }}=0.3 \mu$ bar to $p_{\text {high }}=55 \mu$ bar) at a rate $d T / d t$, a flux of $F=(d T / d t) c_{p}\left(p_{\text {high }}-p_{\text {low }}\right) / g$ has to be conducted away. $F=0.90$ or $0.35 \mathrm{erg} \mathrm{cm}^{-2} \mathrm{~s}^{-1}$ for our nominal or conservative rates of $d T / d t=3$ or $1.2 \mathrm{~K} \mathrm{yr}^{-1}$, respectively, The flux is related to the thermal gradient through $F=\kappa d T / d z$, where $\kappa$ is the thermal conductivity. For $T$ in $\mathrm{K}$ and $\kappa$ in erg $\mathrm{cm}^{-1} \mathrm{~s}^{-1} \mathrm{~K}^{-1}, \kappa=252 T^{0.751}$ (Hanley et al. 1970), implying a thermal gradient of $8.5 \mathrm{~K} \mathrm{~km}^{-1}$ is needed to cool the atmosphere at the nominal rate of $3 \mathrm{~K} \mathrm{yr}^{-1}$, or a gradient of $3.3 \mathrm{~K} \mathrm{~km}^{-1}$ to cool at the conservative rate of $1.2 \mathrm{~K} \mathrm{yr}^{-1}$. This is somewhat higher than the gradients observed by the Voyager UVS (Herbert et al. 1987), which peak at $1.1 \mathrm{~K} \mathrm{~km}^{-1}$ near 1-6 $\mu \mathrm{bar}$, and then decrease to $0.3 \mathrm{~K} \mathrm{~km}^{-1}$ from $6-500 \mu$ bar. This suggests that thermal conduction alone is an unlikely candidate for the responsible cooling mechanism, especially since, as with the radiative cooling argument, we have made a "best case" scenario by ignoring heating sources. However, the UVS thermal profiles underestimated the thermal gradients near $1 \mu \mathrm{bar}$ on Jupiter by a factor of 3 (Festou et al. 1981, Young et al. 1997), so we cannot rule out a similar readjustment here. As on Jupiter, one way to constrain the thermal gradients in this region is by measurements of the temperatures near the homopause from $\mathrm{H}_{2}$ fluorescence (Liu and Dalgarno 1996), in combination with ground-based and UVS occultations (Yelle et al. 1996). 


\subsection{Heating and Cooling by Adiabatic Vertical Motion}

Given the difficulties associated with cooling by radiation or conduction, we turn now to dynamical effects, such as adiabatic cooling. We show below that adiabatic cooling can easily account for the observed heating and cooling rates. Furthermore, the adiabatic cooling may be driven by the momentum deposition of breaking gravity waves. A complete global circulation model would answer specific questions about the feasibility of the proposed cooling mechanism, such as the link between circulation and season, but is beyond the scope of this paper. Instead, we use scaling arguments and simplified dynamical equations, following McLandress (1998).

Local upward adiabatic motion of the Uranian atmosphere causes local cooling, as suggested by Strobel et al. (1991) to explain $200 \mathrm{~K}$ contrasts seen at 1-10 $\mu$ bar, and quantitatively modeled by Flasar et al. (1987) to explain $\sim 2 \mathrm{~K}$ temperature contrasts seen near the tropopause. Any mechanism that depends on adiabatic cooling implies either a global compression or rarefaction of the upper atmosphere, or a corresponding region of adiabatic heating. The regions of corresponding downwelling may be at higher latitudes or at other local times of day.

The results of Section 4.2 suggest that radiative heating and cooling can be ignored to first order, compared with the observed heating or cooling rate. If radiative processes can be ignored, then the vertical wind $W$ required to change the temperature at a rate $d T / d t$ is given by

$$
W=-\frac{R}{N^{2} H} \frac{d T}{d t}
$$

where $R$ is the gas constant, $H$ is the scale height, and $N$ is the Brunt-Väisällä frequency. A nominal temperature decrease of $3 \mathrm{~K} \mathrm{yr}^{-1}$ would require a vertical wind of only $0.016 \mathrm{~cm} \mathrm{~s}^{-1}$.

If the large-scale circulation can be treated as approximately two-dimensional in height and latitude, as it can in the Earth's middle atmosphere, then the continuity equation relates meridional wind $(V)$ to the vertical wind,

$$
\frac{1}{a \cos \theta} \frac{\partial}{\partial \theta}(V \cos \theta)+\frac{1}{\rho} \frac{\partial}{\partial z}(\rho W)=0,
$$

where $a$ is the planetary radius, $\theta$ is the latitude, and $\rho$ is the mass density. If the meridional wind varies on a length scale comparable to the planet radius, and the quantity $\rho W$ varies on a length scale comparable to a scale height, then the magnitude of $V$ can be estimated to be of the order $(a / H) W$, or roughly $8 \mathrm{~cm} \mathrm{~s}^{-1}$. This rough estimate applies to meridional winds that are either inter-hemispheric or symmetric about the equator. If the circulation follows a diurnal pattern, then an analogous continuity equation estimates the deviation of the zonal wind from its zonal mean, with a similar magnitude estimate.
The horizontal momentum equation for the zonal wind can be simplified as

$$
\frac{\partial U}{\partial t}-f V=F_{u},
$$

where $U$ is the zonal wind, $f$ is the Coriolis parameter, and $F_{u}$ is the zonal momentum source. The equation for $\partial V / \partial t$ is similar, and applies if we assume that the circulation is a diurnal pattern. If we assume that the zonal wind changes slowly over a Uranian day, we can ignore the acceleration of zonal wind, and set $\partial U / \partial t=0$. If we evaluate $f$ at a latitude of $45^{\circ}$, then we can estimate that a cooling rate of $3 \mathrm{~K} \mathrm{yr}^{-1}$ can be caused by a zonal momentum source of $0.0016 \mathrm{~cm} \mathrm{~s}^{-2}$. This source is not unreasonably large; the momentum source apparently required to explain the temperatures in the Earth's mesosphere is about $0.1 \mathrm{~cm} \mathrm{~s}^{-2}$ (Andrews et al. 1987, p. 305).

The processes of momentum deposition and adiabatic cooling outlined above is analogous to that which causes the low temperatures in the Earth's summer mesosphere (e.g., Andrews et al. 1987, McLandress 1998). In the Earth's summer mesosphere, the zonal momentum source is driven by the deposition of momentum carried by breaking gravity waves. The Earth is an imperfect analogy for two reasons. First, adiabatic cooling in the terrestrial mesosphere leads to cooler equilibrium temperatures, rather than a time derivative of temperature as in our Eq. (2). Second, the mesospheric circulation on the Earth during solstice is primarily upwelling in the summer hemisphere with downwelling in the winter hemisphere. In contrast, Uranian temperatures increase from 1977 to 1983 at both northern and southern equatorial latitudes (Fig. 5a), suggesting downwelling in both hemispheres. Nevertheless, we can investigate whether similar forcing is expected on Uranus based on observed waves in the upper atmosphere.

To estimate the momentum forcing from breaking gravity waves on Uranus, we turn to parameterizations of gravity wave drag. Gravity waves are, in general, too small-scale to include in global circulation models. The solution is to parameterize the global effect of the interaction of small-scale gravity waves and the mean flow. Several parameterizations successfully reproduce the mesospheric temperatures on the Earth; for the purpose of evaluating the viability of the mechanism on Uranus, we use the Lindzen parameterization (Lindzen 1981). In this simple parameterization, a single wave grows in amplitude until the temperature gradients of the wave are large enough that the wave becomes convectively unstable; at this point, the instability of the wave leads to eddy diffusion that is just large enough to limit the growth of the wave, and maintain gradients within the adiabatic lapse rate. For a saturated wave propagating through a windless background atmosphere, the zonal momentum source

$$
F_{u}=\frac{k c^{3}}{2 N H}=\frac{k N^{2}}{2 H m^{3}},
$$

where $k=2 \pi / L_{\mathrm{h}}$ and $m=2 \pi / L_{\mathrm{z}}$ are the horizontal and 
vertical wavenumbers, $c$ is the horizontal phase speed, and $L_{\mathrm{h}}$ and $L_{\mathrm{z}}$ are the horizontal and vertical wavelengths. We can further relate $k$ and $m$ to the eddy diffusion coefficient $D$,

$$
D=\frac{N}{2 H} \frac{k}{m^{4}},
$$

allowing us to express the forcing in terms of the vertical wavelength of the breaking wave:

$$
F_{u}=D N \frac{2 \pi}{L_{\mathrm{z}}}
$$

Using $D=10^{4} \mathrm{~cm}^{2} \mathrm{~s}^{-1}$ (Atreya et al. 1991, and references therein), we can estimate that breaking gravity waves with vertical wavelengths of $L_{\mathrm{z}}=2.5 \mathrm{~km}$ may be responsible for a cooling rate of $3 \mathrm{~K} \mathrm{~km}^{-1}$ in the Uranian upper atmosphere. The vertical wavelength can also be estimated independently of the eddy diffusion coefficient from the stratification of the gravity waves, as characterized by $L_{\mathrm{h}} / L_{\mathrm{z}}=m / k$. From simultaneous measurements of a 1980 Uranus occultation at sites separated by 25 to $150 \mathrm{~km}$, French et al. (1982) estimate $m / k \approx 60$. Substitution into Eqs. 5 and 6 yields $L_{\mathrm{z}} \approx 11 \mathrm{~km}$ and $D=4.5 \times$ $10^{4} \mathrm{~cm}^{2} \mathrm{~s}^{-1}$.

Waves with vertical wavelengths in the range of 2 to $11 \mathrm{~km}$ have been seen in the upper atmosphere of Uranus. In particular, French et al. (1983) report "variations of a few tenths of a degree Kelvin over an altitude range of a few kilometers." The implied thermal gradient is comparable to the adiabatic lapse rate $\left(g / c_{p}=0.6 \mathrm{~K} / \mathrm{km}\right)$, characteristic of saturated gravity waves. These waves may be generated at lower altitudes. The energy required to generate these waves is $<0.05 \mathrm{erg} \mathrm{cm}^{-2} \mathrm{~s}^{-1}$ (French et al. 1983), a negligible fraction of the incident solar energy. However, the waves seen in Uranus's stratosphere at 10 to 0.4 mbar in Voyager radio occultation data (Hinson and Magalhaes 1991) yield momentum forcing that is an order of magnitude too small to drive the required vertical winds.

The wave-driven adiabatic heating and cooling rates discussed here arise from the interaction of the wave's momentum flux with the background winds. They are distinct from either the direct deposition of the wave energy flux during dissipation, or the cooling that results from turbulent heat flux. The sum of these two competing effects leads to a heating or cooling rate that is proportional to $N^{2} D / 2 c_{p}\left(0.3 \mathrm{~K} \mathrm{yr}^{-1}\right)$, where the constant of proportionality can be either positive or negative, depending on unknown parameters such as the eddy Prandtl number and the vertical variation of eddy diffusion (Strobel et al. 1985, Schoeberl et al. 1983). Therefore, on Uranus as on Earth, waves may affect mesospheric temperatures more through momentum transport than through energy transport.

The discussion in this section is intended only to demonstrate the viability of the dynamical cooling, and is admittedly not selfconsistent. In particular, the estimate of the vertical wavelengths assumed no vertical shear of horizontal winds, even though latitudinal changes in temperature will induce zonal winds through the thermal wind equation. A more complete analysis would require information about the winds in the Uranian middle atmosphere, and the application of a global circulation model.

\section{CONCLUSIONS}

From two probes of the Uranian upper atmosphere in November 6, 1998, we derive equivalent isothermal temperatures of $116.7 \pm 7.9 \mathrm{~K}$ for immersion, and $124.8 \pm 15.5 \mathrm{~K}$ for emersion, indicating that the warming trend observed between 1977 and 1983 has reversed. If interpreted as a purely temporal change, the 1998 observations indicate a cooling rate of $3 \mathrm{~K} \mathrm{yr}^{-1}$. This cooling rate cannot be caused by radiative processes alone. Thus, the observations presented here strongly suggest that there is a nonradiative energy sink in the Uranian upper atmosphere. Possibilities for the sink include thermal conduction to the stratosphere, and adiabatic cooling.

We estimate that thermal conduction, transporting energy from a warm upper atmosphere to a cooler stratosphere, can effectively cool the upper atmosphere if there are thermal gradients near $50 \mu$ bar of $3-10 \mathrm{~K} \mathrm{~km}^{-1}$. The Voyager UVS observations at this pressure argue against such steep gradients.

Alternatively, the cooling can be dominated by dynamics. A vertical wind of $0.016 \mathrm{~cm} \mathrm{~s}^{-1}$ will cause adiabatic cooling at $3 \mathrm{~K} \mathrm{yr}^{-1}$. We argue that a wind of this magnitude can be created by the deposition of momentum by breaking gravity waves with vertical wavelengths of $2-11 \mathrm{~km}$, in a process similar to that which cools the summer mesopause on the Earth. Furthermore, waves of the required wavelength and amplitude have been seen in thermal profiles derived from Uranian occultations.

These two mechanisms can be tested by (i) the combination of $\mathrm{H}_{2}$ airglow spectroscopy and refractive occultations to constraining gradients in the 1-100 $\mu$ bar region (as has been done for Jupiter; see Yelle et al. 1996), (ii) continued observations of Uranian occultations to track the variation of temperatures with season, latitude, and insolation, and (iii) the use of seasonal global circulation models to investigate the interaction of breaking gravity waves with mean winds.

\section{ACK NOW LED G MENTS}

This work was supported, in part, by NASA grants NAGW-3296, NAG5-6826, NAG5-7861, NAG5-4195, and NSF grants AST-9322115 and AST-0073447. Observations at Lowell Observatory's Perkins telescope were obtained as part of a partnership between Boston University and Lowell Observatory, while LAY was a Senior Research Associate at Boston University. Thanks to Floyd Herbert for providing an electronic version of the UVS profile, to John Noble, Bob Millis, and Ken Janes for their help in scheduling these observations at the Perkins telescope, and to Dave Griep for his assistance at the IRTF. The solar F10.7 fluxes were provided by the National Geophysical Data Center, at ftp://ftp.ngdc.noaa.gov/STP/SOLAR_DATA/SOLAR_RADIO/FLUX.

\section{REFERENCES}

Abramowitz, M., and I. A. Stegun 1964. Handbook of Mathematical Functions. Applied Mathematics Series, Vol. 55. National Bureau of Standards; Washington, reprinted 1968, Dover, New York. 
Allen, C. W. 1976. Astrophysical Quantities, 3rd ed. Athlone, London.

Andrews, D. G., J. R. Holton, and C. B. Leovy 1987. Middle Atmosphere Dynamics. Academic Press, Orlando.

Appleby, J. F. 1980. Atmospheric Structures of the Giant Planets from RadiativeConvective Equilibrium Models. Ph.D. thesis, State University of New York at Stony Brook.

Appleby, J. F. 1986. Radiative-convective equilibrium models of Uranus and Neptune. Icarus 65, 383-405.

Appleby, J. F. 1990. $\mathrm{CH}_{4}$ non-local thermodynamic equilibrium in the atmospheres of the giant planets. Icarus $\mathbf{8 5}, 355-379$.

Atreya, S. K., B. R. Sandel, and P. N. Romani 1991. Photochemistry and vertical mixing. In Uranus (J. Bergstralh, E. D. Miner, and M. S. Matthews, Eds.), Univ. of Arizona Press, Tucson.

Baron, R. L., R. G. French, and J. L. Elliot 1989. The oblateness of Uranus at the 1-microbar level. Icarus 78, 119-130.

Baum, W. A., and A. D. Code 1953. A photometric observation of the occultation of $\sigma$ Arietis by Jupiter. Astron. J. 58, 108-112.

Bishop, J., S. K. Atreya, F. Herbert, and P. Romani 1990. Reanalysis of Voyager 2 UVS occultations at Uranus: Hydrocarbon mixing ratios in the equatorial stratosphere. Icarus 88, 448-464.

Broadfoot, A. L., F. Herbert, J. B. Holberg, D. M. Hunten, S. Kumar, B. R. Sandel, D. E. Shemansky, G. R. Smith, R. V. Yelle, D. F. Strobel, H. W. Moos, T. M. Donahue, S. K. Atreya, J. E. Blamont, J. C. McConnell, A. J. Dessler, S. Linick, and R. Springer 1986. Ultraviolet spectrometer observations of Uranus. Science 233, 74-79.

Buie, M. W., R. L. Millis, L. H. Wasserman, J. L. Elliot, S. J. Bus, E. W. Dunham, E. F. Young, W. B. Hubbard, D. M. Hunten, and W. K. Wells 1993. CCD camera occultation system. Bull. Am. Astron. Soc. 25, 1115.

Conrath, B. J., D. Gautier, R. A. Hanel, G. Lindel, and A. Marten 1987. The helium abundance of Uranus from Voyager measurements. J. Geophys. Res. 92, 15003-15010.

Covington, A. E. 1969. Solar radio emission at $10.7 \mathrm{~cm}$. Royal Astron. Soc. Canada 63, 125.

Elliot, J. L., and E. Dunham 1979. Temperature structure of the Uranian upper atmosphere. Nature 279, 307-308.

Elliot, J. L., and L. A. Young 1992. Analysis of stellar occultation data for planetary atmospheres. I. Model fitting, with application to Pluto. Astron. J. 103, 991-1015.

Elliot, J. L., E. Dunham, and D. Mink 1977. The rings of Uranus. Nature 267, 328-330.

Encrenaz, T., B. Schulz, P. Drossart, E. Lellouch, H. Feuchtgruber, and S. K. Atreya 2000a. The ISO spectra of Uranus and Neptune between 2.5 and $4.2 \mu \mathrm{m}$ : Constraints on albedos and $\mathrm{H}_{3}^{+}$. Astron. Astrophys. 358, L83-L87.

Encrenaz, T., B. Schulz, P. Drossart, E. Lellouch, H. Feuchtgruber, and S. K. Atreya 2000b. Erratum: The ISO spectra of Uranus and Neptune between 2.5 and $4.2 \mu \mathrm{m}$ : Constraints on albedos and $\mathrm{H}_{3}^{+}$. Astron. Astrophys. 362, 1174-1180.

Festou, M. C., S. K. Atreya, T. M. Donahue, B. R. Sandel, D. E. Shemansky, and A. L. Broadfoot 1981. Composition and thermal profiles of the Jovian upper atmosphere determined by the Voyager ultraviolet stellar occultation experiment. J. Geophys. Res. 86, 5715-5725.

Flasar, F. M., B. J. Conrath, J. A. Pirraglia, and P. J. Gierasch 1987. Voyager infrared observations of Uranus' atmosphere-Thermal structure and dynamics. J. Geophys. Res. 92, 115011-15018.

French, R. G., J. L. Elliot, and P. Gierasch 1978. Analysis of stellar occultation data. Icarus 33, 186-202.

French, R. G., J. L. Elliot, B. Sicardy, P. Nicholson, and K. Matthews 1982. The upper atmosphere of Uranus-A critical test of isotropic turbulence models. Icarus 51, 491-508.
French, R. G., J. L. Elliot, E. W. Dunham, D. A. Allen, J. H. Elias, J. A. Frogel, and W. Liller 1983. The thermal structure and energy balance of the Uranian upper atmosphere. Icarus 54, 399-414.

French, R. G., T. J. Jones, and A. R. Hyland 1987. The 1 May 1982 stellar occultation by Uranus and the rings Observations from Mount Stromlo Observatory. Icarus 69, 499-505.

French, R. G., J. L. Elliot, L. M. French, J. A. Kangas, K. J. Meech, M. E. Ressler, M. W. Buie, J. A. Frogel, J. B. Holberg, J. J. Fuensalida, and M. Joy 1988. Uranian ring orbits from Earth-based and Voyager occultation observations. Icarus 73, 349-378.

French, R. G., P. D. Nicholson, C. C. Porco, and E. A. Marouf 1991. Dynamics and structure of the Uranian rings. In Uranus (J. Bergstralh, E. D. Miner, and M. S. Matthews, Eds.), Univ. of Arizona Press, Tucson.

Goldsmith, D. W. 1963. Differential refraction in planetary atmospheres with linear scale height gradients. Icarus 2, 341-349.

Hanley, H. J. M., R. D. McCarthy, and H. Inteman 1970. The viscosity and thermal conductivity of dilute gaseous hydrogen from 15 to 5000 K. J. Res. Natl. Bur. Stand. Sec. A. 74, 331.

Herbert, F., B. R. Sandel, R. V. Yelle, J. B. Holberg, A. L. Broadfoot, D. E. Shemansky, S. K. Atreya, and P. N. Romani 1987. The upper atmosphere of Uranus-EUV occultations observed by Voyager 2. J. Geophys. Res. 92, 115093-15109.

Hinson, D. P., and J. A. Magalhaes 1991. Equatorial waves in the stratosphere of Uranus. Icarus 94, 64-91.

Klemola, A. R., and D. J. Mink 1991. Occultations by Uranus and Neptune1991-1999. Astron. J. 102, 389-394.

Lindzen, R. S. 1981. Turbulence and stress owing to gravity wave and tidal breakdown. J. Geophys. Res. 86, 9707-9714.

Liu, W., and A. Dalgarno 1996. The ultraviolet spectrum of the jovian dayglow. Astrophys, J. 462, 502.

Mansfield, C. R., and E. R. Peck 1969. Dispersion of helium. J. Opt. Soc. Am. 59, 199-204.

Marley, M. S., and C. P. McKay 1999. Thermal structure of Uranus' atmosphere. Icarus 138, 268-286.

McLandress, C. 1998. On the importance of gravity waves in the middle atmosphere and their parameterization in general circulation models. J. Atmos. Solar-Terr. Phys. 60, 1357-1383.

Millis, R. L., L. H. Wasserman, and P. V. Birch 1977. Detection of rings around Uranus. Nature 267, 330-331.

Millis, R. L., L. H. Wasserman, and R. G. French 1987. Observations of the 22 April 1982 stellar occultation by Uranus and the rings. Icarus 69, 176-184.

Moses, J. I., E. Lellouch, B. Bézard, G. R. Gladstone, H. Feuchtgruber, and M. Allen 2000. Photochemistry of Saturn's atmosphere. Icarus 145, 166-202.

Peck, E. R., and S. Huang 1977. Refractivity and dispersion of hydrogen in the visible and near-infrared. J. Opt. Soc. Am. 67, 1550-1554.

Rizk, K., and D. M. Hunten 1990. Solar heating of the Uranian mesopause by dust of ring origin. Icarus $\mathbf{8 8 ,} 429-447$.

Rothman, L. S., R. R. Gamache, R. H. Tipping, C. P. Rinsland, M. A. H. Smith, D. C. Benner, V. M. Devi, J.-M. Flaud, C. Camy-Peyret, and A. Perrin 1992. The HITRAN molecular data base-Editions of 1991 and 1992. J. Quant. Spectrosc. Radioact. Trans. 48, 469-507.

Schoeberl, M. R., D. F. Strobel, and J. P. Apruzese 1983. A numerical model of gravity wave breaking and stress in the mesosphere. J. Geophys. Res. 88, 5249-5259.

Sicardy, B., M. Combes, J. Lecacheux, P. Bouchet, A. Brahic, P. Laques, C. Perrier, L. Vapillon, and Y. Zeau 1985. Variations of the stratospheric temperature along the limb of Uranus-Results of the 22 April 1982 stellar occultation. Icarus 64, 88-106.

Snell, C. M., and A. M. Heiser 1968. Air mass determinations from the U.S. standard atmosphere. P.A.S.P. 80, 336-338. 
Standish, E. M., X. X. Newhall, J. G. Williams, and W. M. Folkner 1995. JPL planetary and lunar ephemerides, DE403/LE403. JPL Interoffice Memorandum 314.10-127.

Strobel, D. F., J. P. Apruzese, and M. R. Schoeberl 1985. Energy balance constraints on gravity wave induced eddy diffusion in the mesosphere and lower thermosphere. J. Geophys. Res. 90, 13067-13072.

Strobel, D. F., R. V. Yelle, D. E. Shemansky, and S. K. Atreya 1991. The upper atmosphere of Uranus. In Uranus (J. Bergstralh, E. D. Miner, and M. S. Matthews, Eds.), Univ. of Arizona Press, Tucson.

Tobiska, W. K., and F. G. Eparvier 1998. EUV97: Improvements to EUV irradiance modeling in the soft X-ray and FUV. Solar Phys. 177, 147-159.

Trafton, L. M., S. Miller, T. R. Geballe, J. Tennyson, and G. E. Ballester 1999. $\mathrm{H}_{2}$ quadrupole and $\mathrm{H}_{3}^{+}$emission from Uranus: The Uranian thermosphere, ionosphere, and aurora. Astrophys. J. 524, 1059-1083.
Tyler, G. L., D. N. Sweetnam, J. D. Anderson, J. K. Cambell, V. R. Eshleman, D. P. Hinson, G. S. Levy, G. F. Lindal, E. A. Marouf, and R. A. Simpson 1986. Voyager 2 radio science observations of the Uranian system: atmosphere, rings, and satellites. Science $\mathbf{2 3 3}, \mathbf{7 9 - 8 4}$.

Wasserman, L. H., and J. Veverka 1973. On the reduction of occultation lightcurves. Icarus 20, 322-345.

Wolven, B. C., and P. D. Feldman 1998. Temporal variations in the Jovian dayglow: Hopkins Ultraviolet Telescope spectra from the Astro-1 and Astro-2 missions. Bull. Am. Astron. Soc. 30, 1077.

Yelle, R. V., L. A. Young, R. J. Vervack, R. Young, L. Pfister, and B. R. Sandel 1996. Structure of Jupiter's upper atmosphere: Predictions for Galileo. J. Geophys. Res. 101, 2149-2162.

Young, L. A., R. V. Yelle, R. Young, A. Seiff, and D. B. Kirk 1997. Gravity waves in Jupiter's thermosphere. Science 276, 108-111. 